\title{
Evaluation of SIGMA and SCTPmx for High Handover Rate Vehicle
}

\author{
Hala Eldaw Idris Jubara \\ Faculty of Electrical Engineering \\ Universiti technologi Malaysia \\ Skudai 81310 Johor Malaysia
}

\author{
Sharifah Hafizah Syed Ariffin \\ Faculty of Electrical Engineering \\ Universiti technologi Malaysia \\ Skudai 81310 Johor Malaysia
}

\begin{abstract}
Rapid technological advance in wireless mobile communication offered Internet accessibility at anytime and anywhere including high speed wireless environment such as in high speed trains, fast moving cars etc. However, wireless Quality of Service (QoS) provisioning in such high speed movement is more difficult and challenging to be tackled than in a fixed-wired environment. This paper discussed transport layer protocol, SCTP to support seamless handover that can guarantees and maintain high QoS in high speed vehicle. The comparison of the selected protocol have been identified and analysed in two handover techniques SIGMA and SCTPmx to optimize its performance in high speed vehicular environment.
\end{abstract}

Keywords-handover latency; speed; SCTP; mobility management; vehicle.

\section{INTRODUCTION}

The need for host mobility support in the Internet is increasing with the explosion of wireless and mobile communication devices. Therefore, network layer solutions (such as Mobile IP) are common to support host mobility in the Internet. Network layer solutions however have their own drawbacks, such as the need of support from the network (Home/Foreign Agents), triangular routing, long handoff latency, etc. Moreover, network layer solutions hide the mobility from the transport protocols, where the flow and congestion control tasks are performed, which Impedes performance optimization. Transport layer solutions on the other hand allow the transport protocol to be aware of mobility and hence adjust changes in the path characteristics when required. Therefore, transport layer could be a better place to tackle the host mobility problem in the Internet [1][2][4][17][18][21]. However, transport layer mobility solution needs to have the following functionalities: Movement Detection, Handoff Management, and Location Management [3].

High-speed transportations; e.g., vehicle along highway are more usable around rapid growth of technologies and needs to maintain service continuity (e.g. real-time and delay-sensitive applications). In fact [2][18][22], making mobility seamless as the movement speed of vehicle increases is more challenging. Mobile users are expecting to receive similar services as fixed users at home or office. This work suppose a scenario of vehicle moving along highway with high speed up to $120 \mathrm{~km} / \mathrm{hr}$, and perform horizontal handover between WiMAX base stations (BS) because it is more frequent type of handover in daily usage, and using single interface is less power consumption and hardware.

The rest of the paper is organized as follows. Section II shows the related works. An overview of Mobility management in highly mobile vehicle communicate to infrastructure (V2I) in terms of five requirements is mentioned in section III. The selected two techniques to be compared related to the problem statement are well-defined in section VI respectively. Section V describes simulation topology and parameters. Section IV presents Handover latency comparison results of the selected techniques mentioned, and section IIV conclude the paper.

\section{RELATED WORK}

In high speed vehicles scenarios the needs for network communication for interactive and real-time applications is becoming gradually more important. Therefore many seamless mobility approaches have been developed. The intent is to avoid service disruption and minimize the awareness of service degradation while mobile device is moving fast and changing the point of attachment from one access point to another. References [2][14] and [15] described various approaches that can support seamless and lossless handover in high speed transportation system. Work in [2] exploit prediction technique is proposed to improve and optimize its performance in high speed environment. Thus, there would be no problem regarding insufficient time in connection establishment as the movement speed increase. A study shows in [15] also suggest 802.21 centric approaches to exploit a prior knowledge method where network information is gathered from both mobile terminal and network infrastructure to establish an earlier connection with new subnet. in order to reduce the effect of service interruption in high movement speed environment. Another research presented in [14] propose a packet forwarding control (PFC) scheme to select a common ahead point (CAP) as the tunnel source to forward packets. So that packets can be sent through a shorter delivery path during handover. Author in [19] proposed Network Mobility Protocol for Vehicular Ad Hoc Networks. They proposed NEMO protocol for VANET in highway, since every car is moving in a fixed direction with high moving speed, the car adopting our protocol can acquire IP address from the VANET through vehicle to vehicle communications. In [19]they presents a cross-layer fast handover scheme, called vehicular fast handover scheme 
(VFHS), where the physical layer information is shared with the MAC layer, to reduce the handover delay. All of above mention works focus for the handover in lower layer (L2, L3) and the transportation layer will not be aware of handover and may cause packet loss.

\section{Mobility Management for Highly Mobile Vehicular NETWORKS}

Mobility management in vehicular networks should guarantee the reachability to correspondent nodes $(\mathrm{CN})$ in the Internet as well as the global reachability to mobile nodes in a vehicular network. Therefore, the mobility management has to meet the following requirements [4].

\section{A. Seamless mobility}

Mobility of vehicles should be seamless regardless of vehicle's location and wireless technology. Also, accessibility and service continuity should be guaranteed.

\section{B. Fast Handover}

Fast handover is needed for delay sensitive ITS applications (e.g., safety, internet access, etc.). Fast handover is also a crucial requirement for wireless networks with small coverage area (e.g., WiFi network), since the vehicle with high speed spends short period of time at each point of attachment (e.g., Base station), consequently high handover rate.

\section{IPv6 support}

The global reachability requires a globally reliable routable IP address for each mobile node. IPv6 with large address space can support a unique address for each mobile device in the vehicles. In addition, IPv6 also has better support of security and quality of service (QoS) which are the necessary requirements of ITS applications.

\section{High mobility speed}

The Internet access is expected to be constantly connected regardless of the movement speed. It is highly desirable to make these contents available and reliable regardless of time, place, fixed or mobile. As the speed of vehicle increases, the successful probability of handover decreases. Moreover, successful probability of handover is reduced by the same reason as the handover execution time is increased.

\section{E. Movement detection}

The vehicle needs to detect the availability of different types of access networks (e.g., WiMAX base station) known as data link layer handover (L2), and obtain addresses in these networks for communication.

\section{COMPARISON OF SIGMA \& SCTPMX IN HIGH HANDOVER RATE VEHICLE}

\section{A. SIGMA}

SIGMA proposed to design a new scheme for supporting low latency and low packet loss mobility called Seamless IP diversity based Generalized Mobility Architecture (SIGMA). SIGMA uses SCTP; however it can be used with other protocols that support IP diversity. It can also cooperate with normal IPv4 or IPv6 infrastructure without the support of Mobile IP [5][6][7][8][9]. Figure.1 shows the timming diagram of SIGMA. General analysis of factors that affect handover latency of SIGMA described as follows.

\section{1) Seamless mobility}

SIGMA needs to setup a location manager which is not restricted to the same subnet as MN's home network. Using location manager make vehicle movement seamless from upper layers (e.g., no need of home agent). This will make the deployment of SIGMA much more flexible than other protocols as MIP.

\section{2) Handover latency}

SIGMA experienced a seamless handover because it could prepare the new path in parallel with data forwarding over the old path. Therefore, SIGMA can achieve a low handover latency, low packet loss rate and high throughput.

\section{3) IPv6 support}

SIGMA can be used with other protocols that support IP diversity. It can also cooperate with normal IPv4 or IPv6 infrastructure without the support of Mobile IP. The reason is that $\mathrm{MH}$ can use any unique information as its identity such as home

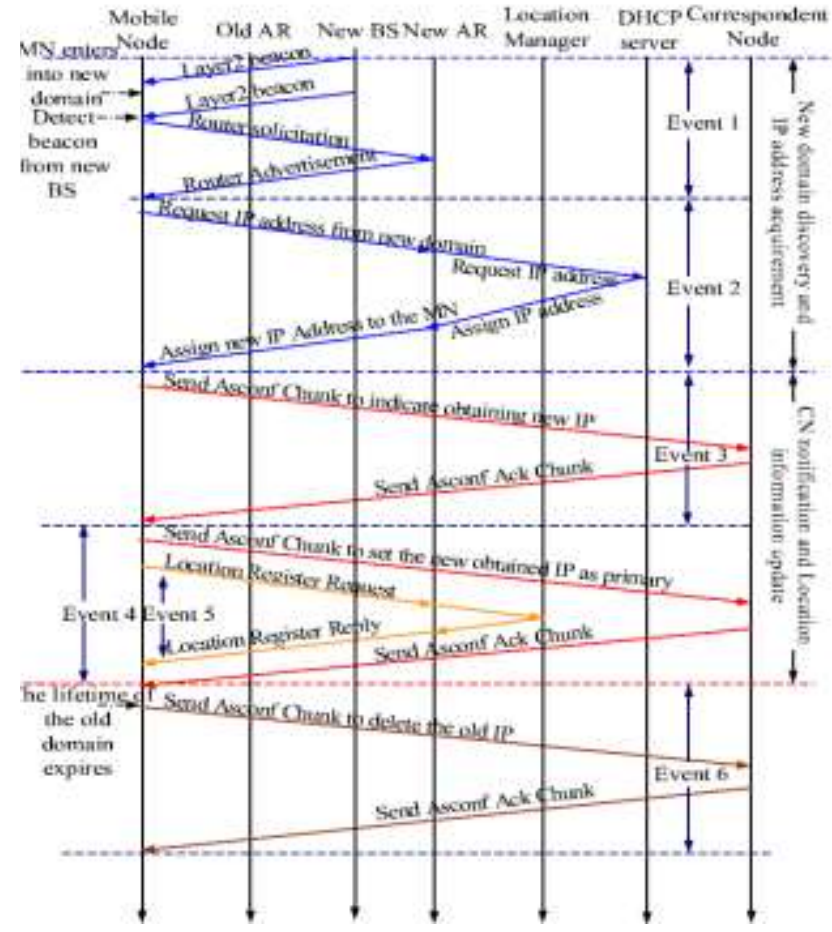

Fig.1 SIGMA handover procedure

address like MIP, or domain name, or a public key defined in Public Key Infrastructure (PKI).

\section{4) High mobility speed}

Because SIGMA do not rely on the assumption that detection of the new agent is well in advance of higher handover latency due to shorter time to prepare for the handover. Also, there is higher possibility that packets are forwarded to the outdated path and get lost; therefore the time instant that $\mathrm{MN}$ can receive packets from the new path will be postponed, and the handover latency increases accordingly. 


\section{5) Movement detection}

SIGMA always requires updating $\mathrm{CN}$ before packets can be received from the new path. Therefore, the increase of this link delay will increase the handover latency (up to $109 \mathrm{~ms}$ in the case of 200ms delay between $\mathrm{CN}$ and PAR).

\section{6) Drawbacks of SIGMA}

- In mobile systems such as IEEE 802.11, GPRS, UMTS, etc. there exists layer 2 handover/ setup latency, which is due to the physical and/or link layer limitations. The SIGMA signaling messages will experience an extra delay, which may break the parallelism that supposed to achieve with IP diversity.

- When MN moves into a new IP domain, it requires some time for MN to obtain a new IP address through DHCP, DHCPv6, or IPv6 Stateless Address Auto-configuration (SAA) [8]. Until this process is finished, MN cannot perform any SIGMA signaling.

- If MN's moving speed is too high, there is no time for $\mathrm{MN}$ to prepare for the new path; the parallelism that can be achieved by IP diversity will be broken. Therefore, the handover performance of SIGMA may affected by these factors mentioned above, even though SIGMA does not require any change on the layer 2 or layer 3 implementation.

\section{B. SCTPmx}

SCTPmx employs a cross-layer control information exchange system between L2, L3 and L4 primitives called LIES to predict handover figure.2. Prior to handover SCTPmx can generate a new address that will be used after handover and can execute duplicate address detection of IPv6 [1].

\section{1) Handover latency}

In SCTPmx, completion of Link layer (L2) handover is immediately reported to the network layer (L2) and the transport layer (L4) by cross layer approach. In addition, duplicate address detection DAD is completed prior to the handover by fast DAD. Thus, the handover latency of SCTPmx is lower than SIGMA in predicting handover.

\section{2) Seamless mobility}

The multihoming mechanism of SCTP can support mobility. Therefore, SCTPmx employs a cross-layer architecture called CEAL to predict handover. This cross-layer allows information exchange between arbitrary layer (L2, L3, and L4) to achieve efficient SCTP communication.

\section{3) IPv6 support}

It observed that SCTPmx can support IPv6, the reason that it has many network layer primitives to achieve efficient TCP communication in a mobile environment through different infrastructure.

\section{4) High vehicle speed}

In SCTPmx handover latency is approximately $100 \mathrm{~ms}$. Thus, for high speed vehicle accordingly high handover rate it cannot receive packets for $100 \mathrm{~ms}$ handover delay and then communication continue for 5999.9ms (WiMAX BS Dwell time*). Therefore, the throughput of SCTPmx is much better of an environment where consecutive handover occurs resulting of high MN speed.

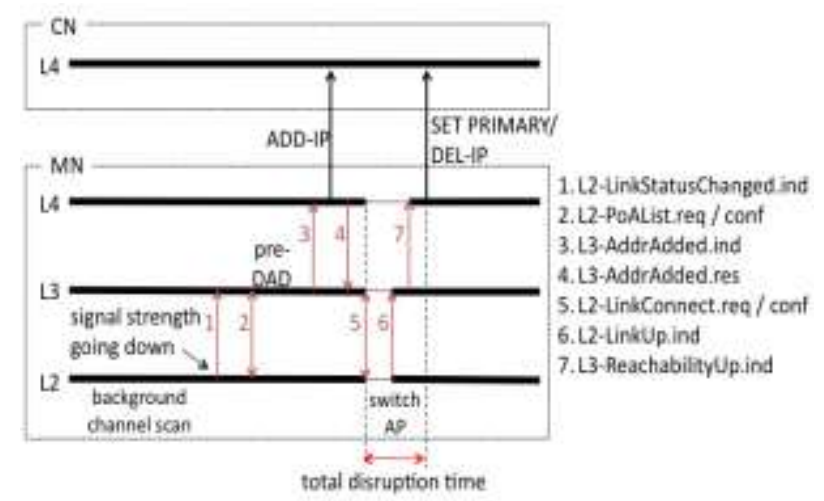

Fig. 2 Handover procedure of SCTPmx

\section{5) Movement detection}

To reduce L2 handover of the vehicle to search for the best base station (BS) to switch when the communication quality is getting worse, SCTPmx introduces the selective background scan procedure. Also, by extending router advertisement (RA) to include options of channel number and send to network layer to learn about the channel number in adjacent cell. Thus, the network layer of the $\mathrm{MN}$ can decide the best access point to handover without channel scan the signal quality to the current access point is going down.

\section{SimUlation TOPOLOGY AND PARAMETERS}

Using SCTP as an illustration is shown in Fig.3, where the vehicle is multi-homed node connected through four wireless access networks (WiMAX BS). Each 2 BSs connected to AR. The WiMAX BS has a radio coverage area of approximately 2000 meters in radius. The overlapping region between two ARs is 200 meters. The correspondent node $(\mathrm{CN})$ is a singlehomed node sending traffic to vehicle, which corresponds to the services like file downloading or web browsing by mobile users and Location Manager (LM) uses by SIGMA.

\section{HANDOVER LATENCY COMPARISON RESULTS}

Handover latency as the time interval between the last data segment received through the old path and the first data segment received through the new path from $\mathrm{CN}$ to $\mathrm{MH}$. In this section, we will examine the impact of different parameters on the overall handover latency of SIGMA and SCTPmx. For handover analysis, we consider the single-homing vehicle that can only use a single network interface at a time. This scenario could be applied to the horizontal handover of an $\mathrm{MN}$ that is moving within homogenous networks. In this case, the link-up of a new link and link-down of the old link will occur at the same time in the underlying link and network layers. That is, the SCTP handover will occur together with the link layer handover at the same time.

For the single-homing $\mathrm{MN}$, the handover latency $\mathrm{T}_{\mathrm{HO} \text {-latency }}$ can be calculated by summing up the time $T_{D H C P}$ (for the configuration of a new IP address from a DHCP server), the time $T_{A S C O N F}$ (for the configuration of a new IP address from a DHCP server), the time $\mathrm{T}_{\mathrm{ASCONF}}$ (for the Add-IP and PrimaryChange and Delete-IP operations in the SCTP handover), and the time $T_{L 2}$ (for the handover at the link layer). Accordingly, the total handover latency of SCTP will be: 


$$
T_{H O}=T_{A S C O N F}+\mathrm{T}_{\mathrm{L} 2}+\mathrm{T}_{\mathrm{DHCP}}
$$

\section{A. Impact of L2 handover}

Handover latency of SIGMA is very low at $10 \mathrm{~ms} \mathrm{L2}$ delay, however, in high speeds vehicle SIGMA experience more handover delay (section IV.a.4) as appear in figure 4. In contrast, SCTPmx employs the selective background scan during data communication and L3 can decide next BS without waiting L2 channel scan.

\section{B. Impact of L3 handover}

SCTPmx uses fast DAD procedure to complete DAD prior to handover. According to RA L3 knows the IPv6 prefix of the next BS and generate the new IPv6 address before handover. Then L3 send fast DAD request to NAR. Therefore, L3 handover latency of SCTPmx is much lower than mSCTP that uses in SIGMA, figure 4[1][6][7][8][9].

On other hand, triangle routing means the packets between $\mathrm{CN}$ and $\mathrm{MH}$ must be routed along a triangular path longer than the optimal one especially in higher speed vehicle [14], which definitely introduces higher latency and high network load in MIP.

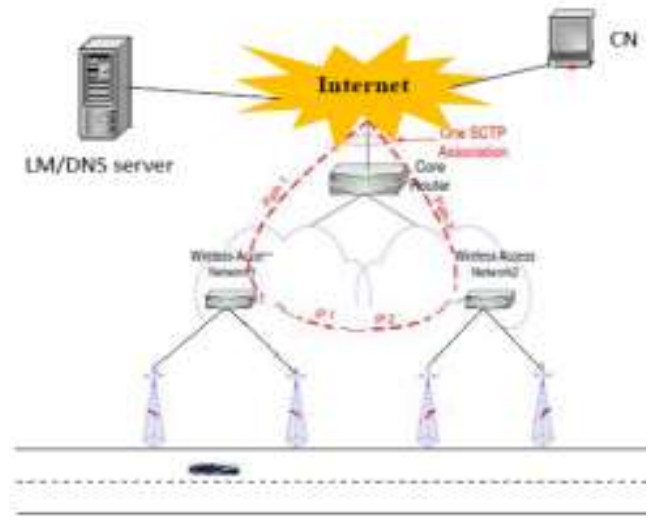

Fig.3 network scenario

In SIGMA, there is no triangle routing because the $\mathrm{CN}$ always sends the packets directly to the MH's current IP address.

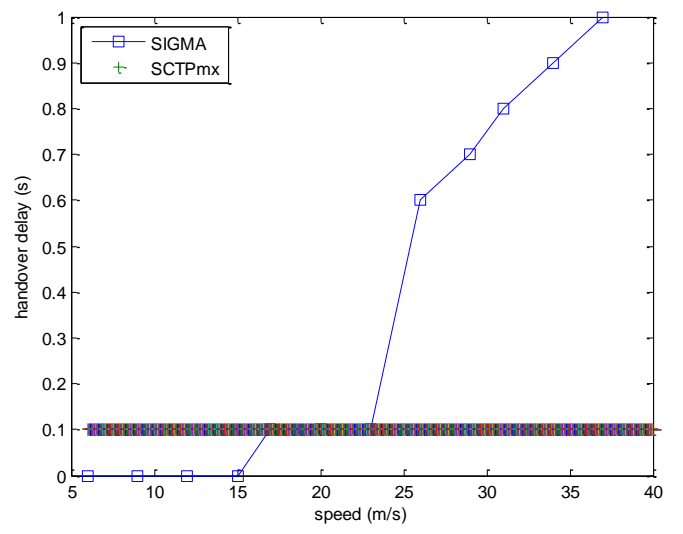

Fig.4 SIGMA \& SCTPmx handover latency for. L2 \& L3

\section{c. Impact of moving speed}

For SCTPmx high moving speed doesn't affect handover and throughput, because handover delay is very low compare to SIGMA protocol that affect by high speed vehicle as shown in figure $4 \& 5$.

Fig.5 comparison of SCTPmx and SIGMA handover for different speeds

\section{CONCLUSIONS}

In this paper we have compared two approaches, SIGMA and SCTPmx, that using SCTP to support mobility in transportation layer in order to realize seamless handover in high speed environment. We have also discussed some of the method used in several approaches that promises seamless handoff and maintain the network quality in such rapidly moving environment.

The evaluation and analysis of these approaches shows the outstanding and drawbacks of each other in case of high

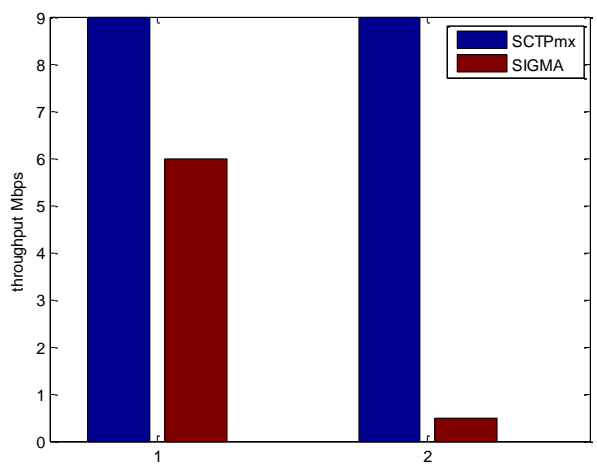

mobility vehicles.

Fig.5 comparison SCTPmx and SIGMA throughputs for different speeds (15$40 \mathrm{~m} / \mathrm{s})$.

\section{ACKNOELEDGEMENT}

The support of my supervisor and others is gratefully acknowledged. This work was supported by mosti grant.

\section{REFERENCES}

[1] Y. Han, F. Teraoka, "SCTPmx: An SCTP Fast Handover Mechanism Using a Single Interface Based on a Cross-Layer Architecture". IEICE Transactions, 2009: 2864 2873.

[2] N.Yaakob,F.Anwar, "Seamless Handover Mobility Schemes over High Speed Wireless Environment", International Conference on Electrical Engineering and Informatics, Indonesia, June 17-19, 2007.

[3] I. Aydin, C.C. Shen, "Evaluating Cellular SCTP over One-Hop Wireless Networks", Mar 2007.

[4] K.Zhu et al, "Mobility and Handoff Management in Vehicular Networks: A Survey", Wieley InterScience, Communication and Mobile Computing, , pp.1-20, 2009.

[5] S.K.Sivagurunathan et al, "Experimental Comparsion of Handoff Performance of SIGMA and Mobile IP", Workshop on High Performance Switching and Routing, HPSR. 2005.

[6] S. Fu, M.Atiquzzaman, "Survivability evaluation of SIGMA and mobile IP", Springer, Wireless Communicatio,2007.

[7] S. Fu, M.Atiquzzaman, "Architecture and Performance of SIGMA: A Seamless Mobility Architecture for Data Networks", IEEE International Conference on Communications, vol.5, pp. 3249 - 3253, May 2005.

[8] S. Fu, M.Atiquzzaman, "Handover latency comparison of SIGMA, FMIPv6, HMIPv6, and FHMIPv6", IEEE GLOBECOM proceeding, Vol. 6, pp. 3809 - 3813, January 2006.

*Dwell time is the time to cross base station coverage area 
[9] P.Chowdhury, S.Reaz, T.Chun Lin, M.Atiquzzaman "Design Issues for SIGMA: Seamless IP diversity based Generalized Mobility Architecture", Technical Report, Feb 2006.

[10] D-Phil Kim and S. Koh, "Analysis of Handover Latency for Mobile IPv6 and mSCTP", Journal of Information Processing Systems, Vol.4, No.3, pp.87-96, September 2008.

[11] D.Phil, S.Joo K, and S.W, “Analysis of SCTP Handover by Movement Patterns", Springer-Verlag Berlin Heidelberg, pp. 521 - 529, 2005.

[12] Y.HAN and F. TERAOKA, "SCTPfx: A Fast Failover Mechanism Based on Cross-Layer Architecture in SCTP Multihoming", AINTEC'08, pp.113-122, Nov 2008, Bangkok, Thailand.

[13] M.Ratola, "Which Layer for Mobility? - Comparing Mobile IPv6, HIP and SCTP", Seminar on Internetworking, April 2004.

[14] C.Ming ,M.Shu and Tz-Heng, "PFC: A packet forwarding control scheme for vehicle handover over the ITS networks", Computer Communications, pp.2815-2826, 2008.

[15] Q.Bouland, W.Yao, Z.Niu and X.Fu,"Optimized FMIPv6 Using IEEE 802.21 MIH Services in Vehicular Networks", Members, IEEE transactions on vehicular technology, nov 2007.

[16] F.TERAOKA, Y.HAN, "Fast handover and fast failover mechanisms based on cross-layer collaboration among the link layer, the network layer and the transport layer", Infocommunications journal,volume LXV, pp10-14, 2010/I.

[17] M. Chang, M.Lee, H.Lee, Y. Hong, and J. Park, "An Enhancement of Transport Layer Approach to Mobility Support", Information Networking. Convergence in Broadband and Mobile Networking, Volume 3391/2005, pp864-873, 2005.

[18] J.Fitzpatrick, S.Murphy, M. Atiquzzaman, J.Murphy, "Using cross-layer metrics to improve the performance of end-to-end handover mechanisms", Computer Communications, Vol.32 pp1600-1612, 2009.

[19] Y.S. Chen and C.H. Cheng, "Network Mobility Protocol for Vehicular Ad Hoc Networks", Wireless Communications and Networking Conference, WCNC, IEEE pp 1-6, April 2009.

[20] K. Chiu, R. Hwangay and Y. Chen, "Cross-Layer Design Vehicle-Aided Handover Scheme in VANETs", wireless communications and mobile computing, pp.1-13, 2009.

[21] M.Chang, M.Lee, H. Lee, Y. Hong and J. Park, "An Enhancement of Transport Layer Approach to Mobility Support", Springer-Verlag Berlin, pp.864-873, 2005.

[22] Abd. Ezzouhairi, Al. Quintero and S. Pierre," adaptive end-to-end mobility scheme for seamless horizontal and vertical handoffs", Ubiquitous Computing and Communication Journal, pp.1-14, 2009. 